\title{
Protocol for Urgent and Emergent Cases at a Large Academic Level 1 Trauma Center
}

Karim Ahmed ${ }^{1}$, Corinna Zygourakis ${ }^{1}$, Sammy Kalb ${ }^{2}$, Zach Pennington ${ }^{1}$, Camilo Molina ${ }^{1}$, Terry Emerson 3 , Nicholas Theodore ${ }^{1}$

1. Neurosurgery, Johns Hopkins Hospital, Baltimore, USA 2. Neurosurgery, Barrow Neurological Institute, Phoenix, USA 3. Surgery, Johns Hopkins Hospital, Baltimore, USA

Corresponding author: Corinna Zygourakis, corinna.zygourakis@gmail.com

\section{Abstract}

\section{Background}

Level 1 trauma centers are capable of caring for every aspect of injury and contain 24-hour in-house coverage by general surgeons, with prompt availability of nearly all other disciplines upon request. Despite the wide variety of trauma, currently reported protocols often focus on a single surgical service and studies describing their implementation are lacking. The aim of the current study was to characterize all urgent and emergent cases at a large academic Level 1 trauma center, characterize the specialty and nature of emergent operative cases, and assess the efficacy of the institutional trauma protocol on timing of surgery.

\section{Methods}

For this retrospective review, all urgent and emergent cases treated at a single institution, during a 34month period (January 1, 2015-October 31, 2017), were identified. All included cases were subject to the Institutional Guidelines for Operative Urgent/Emergent Cases. Demographic characteristics for non-elective surgical emergent cases were compiled by level of urgency and operating room (OR) waiting times were compared by year, department, and Level.

\section{Results}

A total of 11,206 urgent and emergent operative cases were included, among over 16 surgical departments. Level 2 cases represented the majority of urgent/emergent cases (33\%-36\%), followed by Level $3(25 \%-26 \%)$, Level 1 (21\%-22\%), Level $4(12 \%-16 \%)$, and Level $5(2 \%-4 \%)$. Univariate analysis demonstrated that the proportion of urgent and emergent cases, by level of urgency, did not significantly differ between each year. Operating room waiting time decreased significantly over each year from 2015, 2016, and 2017: 193.40 $\pm 4.78,177.20 \pm 3.29$, and $82.01 \pm 2.98$ minutes, respectively.

\section{Conclusions}

Received 11/30/2018 Review began 12/17/2018 Review ended 01/19/2019 Published 01/28/2019

\section{() Copyright 2019}

Ahmed et al. This is an open access article distributed under the terms of the Creative Commons Attribution License CC-BY 3.0., which permits unrestricted use, distribution, and reproduction in any medium, provided the original author and source are credited.
To the authors' knowledge, this is the first study to characterize all urgent and emergent cases at a large academic Level 1 trauma center, outline the specialty and nature of emergent operative cases, and assess the efficacy of the institutional trauma protocol on surgical waiting times over a 34-month period.

Categories: Emergency Medicine, Neurosurgery, Quality Improvement

Keywords: urgent surgery, emergency surgery, level 1 trauma center, trauma surgery, tertiary care center

\section{Introduction}

The American Trauma Society and American College of Surgeons (ACS) designate a Level 1 trauma center as one capable of caring for every aspect of injury and containing 24-hour in-house coverage by general surgeons, with prompt availability of orthopedic surgery, neurosurgery, anesthesiology, emergency medicine, radiology, internal medicine, plastic surgery, oral and maxillofacial surgery, pediatric and critical care [1]. There is well-established literature, specific to various surgical specialties, describing the nature of cases classified as urgent/emergent, and the optimal timing for such cases [2-6]. Treatment delays for emergency surgery also significantly increase the economic impact of care, due largely to complications and length of hospital stay [7].

However, there is a lack of descriptive studies that outline all urgent and emergent cases seen at a Level 1 trauma center, identifying the most represented surgical departments and type of cases. Additionally, there is a lack of reported standardized triage protocols that take into consideration appropriate timing for such non-elective urgent cases. Current triage protocols that have been reported in the literature [8-10] underrepresent the variety of surgical specialties present in a Level 1 trauma center, and studies demonstrating their implementation and efficacy are limited. The aim of the current study was to summarize all urgent and emergent cases at a large academic Level 1 trauma center, characterize the 
specialty and nature of emergent operative cases, and assess the efficacy of the institutional trauma protocol on timing of surgery.

\section{Materials And Methods \\ Institutional guidelines for operative urgent/emergent cases}

In an effort to improve waiting times for urgent/emergent surgical cases, and ensure appropriate care for all patients, the Institute for Healthcare Optimization (IHO) was consulted to assist in designing a set of institutional guidelines for the triage of surgical cases. This group has previously applied variable methodology (VM), a concept of appropriating limited resources accounting for variability in healthcare delivery and acuity, successfully in numerous hospitals and institutions [5,11]. An Executive Steering Committee, Advisory Committee, and Working Committee - each consisting of clinicians, hospital administrators, and consultants - were assembled. In combination with variable methodology, queuing theory is an accurate tool to determine the expected supply of a hospital resource and its allocation [12].

Urgent/emergent cases were defined by the institution as patients requiring access to the operating room (OR) within 24 hours of the decision to operate. Clinical need was further classified into five levels, based on the maximum clinically acceptable waiting time between a case being posted and OR access: patient needing surgical intervention within one hour (Level 1), within two hours (Level 2), within six hours (Level 3), within 12 hours (Level 4), and within 24 hours (Level 5). The OR guidelines for the management of urgent/emergent patient flow have been summarized for this institution (Figure 1). An urgent/emergent case is first posted by the treating surgeon, and the patient is prepared for surgery (i.e., NPO, consented, diagnostic workup, surgical site marked). Cases are assigned to an OR and started by level of urgency. Cases within a level are accommodated in the order of posting. The posting surgeon may request a change in the queue within a level or change in the level only if the clinical status of the patient has changed. For urgent/emergent cases that cannot be placed in an OR within the maximal clinically acceptable waiting time, elective cases on the same surgical service are delayed to accommodate the urgent/emergent case. If there are no appropriate elective cases of the same service, then the first available OR of any surgical service is delayed to accommodate the urgent/emergent case. Monthly reports detailing performance regarding maximal clinically acceptable waiting time are distributed to chiefs of each surgical service and reviewed by the institution's Surgical Executive Committee. 


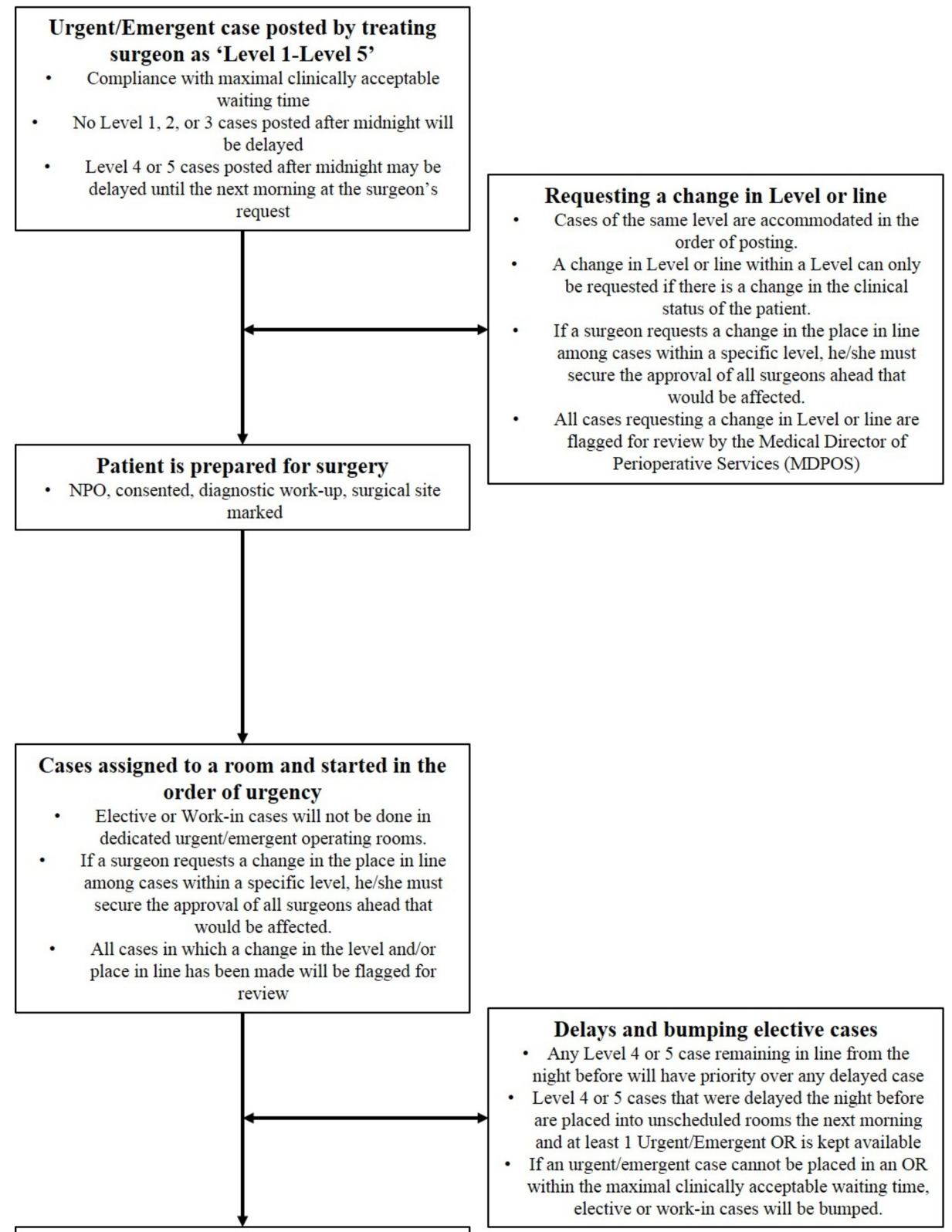

Once a case is assigned to a room, the surgeon must be prepared to operate without delay

- If the surgeon is not ready, the case will go to the bottom of the line for that respective level and the next patient for that level of urgency will be served

\section{FIGURE 1: Flow chart for determination of surgical leveling.}

Flow diagram describing operating room (OR) guidelines for the management of urgent/emergent case flow at a single institution.

\section{Study design and recorded data}

For this retrospective review, all urgent and emergent cases treated at a single institution, the Johns Hopkins Hospital, during a 34-month period (January 1, 2015-October 31, 2017), were identified. All included cases were subject to the Institutional Guidelines for Operative Urgent/Emergent Cases. Operative cases during the study period that were not urgent/emergent were excluded from this study.

Demographic characteristics for urgent cases were compiled by the OR nurse administrator including: the level of urgency (based on institutional guidelines and designated by the treating surgeon), time the case was posted, time the case entered the OR, time the case exited the OR, title of the case, current procedural terminology (CPT) code, surgical specialty, and duration of surgery. The in-room time was calculated as the 
difference between the time a case exited and entered the operating room. The difference between the time a case was posted and entered the operating room was defined as the waiting period. No protected health information or patient information was collected in this study and Institutional Review Board approval was not required since this was conducted as part of a quality improvement initiative.

\section{Statistical analysis}

Continuous demographic data are presented as means with standard deviations. Where applicable, frequencies were compared with Chi-squared tests. A Mann-Whitney U-test was used to compare continuous variables. All analyses were performed in GraphPad Prism 6 (GraphPad Software Inc., La Jolla, California).

\section{Results}

Urgent/emergent cases treated at a large academic Level 1 trauma center over a 34-month period (January 1 , 2015-October 31, 2017) were identified, resulting in the inclusion of 11,206 cases (Table 1). Level 2 cases represented the majority of urgent/emergent cases (33\%-36\%), followed by Level 3 (25\%-26\%), Level 1 (21\%-22\%), Level $4(12 \%-16 \%)$, and Level $5(2 \%-4 \%)$ (Table 1). Chi-square analysis demonstrated that the proportion of urgent and emergent cases, by level of urgency, did not significantly differ between each year $(p>0.05)$ resulting in a similar distribution of Level 1 to Level 5 cases.

\begin{tabular}{|c|c|c|c|c|c|c|c|c|c|c|c|c|c|c|c|}
\hline \multirow{2}{*}{ Department } & \multicolumn{3}{|l|}{ Level 1} & \multicolumn{3}{|c|}{ Level 2} & \multicolumn{3}{|c|}{ Level 3} & \multicolumn{3}{|l|}{ Level 4} & \multicolumn{3}{|c|}{ Level 5} \\
\hline & 2015 & 2016 & $2017^{*}$ & 2015 & 2016 & 2017 & 2015 & 2016 & 2017 & 2015 & 2016 & 2017 & 2015 & 2016 & 2017 \\
\hline Anesthesiology/Pain Medicine & 1 & 0 & 2 & 1 & 0 & 1 & 1 & 1 & 2 & 0 & 22 & 27 & 0 & 1 & 2 \\
\hline Cardiothoracic Surgery & 86 & 85 & 57 & 74 & 79 & 51 & 27 & 17 & 10 & 16 & 17 & 12 & 11 & 10 & 8 \\
\hline Gastroenterology & 11 & 8 & 6 & 54 & 21 & 22 & 6 & 12 & 22 & 0 & 4 & 15 & 0 & 1 & 2 \\
\hline General Surgery & 91 & 101 & 63 & 153 & 150 & 122 & 272 & 209 & 152 & 217 & 255 & 253 & 92 & 27 & 20 \\
\hline Interventional Radiology & 0 & 1 & 0 & 2 & 1 & 0 & 8 & 3 & 0 & 24 & 7 & 1 & 0 & 0 & 0 \\
\hline Neurosurgery & 149 & 150 & 135 & 197 & 166 & 207 & 164 & 128 & 139 & 27 & 41 & 19 & 7 & 6 & 3 \\
\hline Obstetrics/Gynecology & 31 & 41 & 36 & 37 & 52 & 45 & 12 & 19 & 8 & 1 & 4 & 0 & 1 & 2 & 0 \\
\hline Ophthalmology & 0 & 2 & 2 & 14 & 11 & 8 & 8 & 15 & 23 & 7 & 11 & 11 & 2 & 2 & 2 \\
\hline Orthopaedic Surgery & 24 & 27 & 29 & 111 & 90 & 85 & 192 & 217 & 194 & 133 & 187 & 144 & 24 & 15 & 7 \\
\hline Otolaryngology & 105 & 87 & 75 & 78 & 64 & 63 & 51 & 51 & 44 & 18 & 25 & 20 & 7 & 5 & 2 \\
\hline Plastic Surgery & 17 & 20 & 14 & 53 & 40 & 31 & 83 & 69 & 47 & 7 & 22 & 4 & 6 & 4 & 1 \\
\hline Transplant/Abdominal Surgery & 52 & 40 & 33 & 287 & 273 & 193 & 6 & 15 & 11 & 0 & 2 & 1 & 4 & 4 & 2 \\
\hline Trauma Surgery & 171 & 230 & 175 & 222 & 233 & 182 & 120 & 146 & 140 & 2 & 6 & 1 & 1 & 1 & 1 \\
\hline Urology & 26 & 30 & 38 & 71 & 55 & 46 & 45 & 38 & 18 & 16 & 12 & 11 & 6 & 2 & 1 \\
\hline Vascular Surgery & 75 & 42 & 36 & 82 & 57 & 55 & 44 & 36 & 35 & 5 & 3 & 3 & 0 & 0 & 1 \\
\hline Other & 6 & 5 & 4 & 13 & 13 & 14 & 11 & 17 & 24 & 20 & 22 & 21 & 15 & 8 & 4 \\
\hline Total & 845 & 869 & 705 & 1449 & 1305 & 1125 & 1050 & 993 & 869 & 493 & 640 & 543 & 176 & 88 & 56 \\
\hline Proportion of Cases by Level & \multicolumn{3}{|c|}{ Level 1} & \multicolumn{3}{|l|}{ Level 2} & \multicolumn{3}{|l|}{ Level 3} & \multicolumn{3}{|l|}{ Level 4} & \multicolumn{3}{|c|}{ Level 5} \\
\hline 2015 & \multicolumn{3}{|l|}{0.21} & \multicolumn{3}{|l|}{0.36} & \multicolumn{3}{|l|}{0.26} & \multicolumn{3}{|l|}{0.12} & \multicolumn{3}{|l|}{0.04} \\
\hline 2016 & \multicolumn{3}{|l|}{0.22} & \multicolumn{3}{|l|}{0.33} & \multicolumn{3}{|l|}{0.25} & \multicolumn{3}{|l|}{0.16} & \multicolumn{3}{|l|}{0.02} \\
\hline 2017 & \multicolumn{3}{|l|}{0.21} & \multicolumn{3}{|l|}{0.34} & \multicolumn{3}{|l|}{0.26} & \multicolumn{3}{|l|}{0.16} & \multicolumn{3}{|l|}{0.02} \\
\hline
\end{tabular}

TABLE 1: Breakdown of number of cases by level, year, and department.

Distribution of urgent/emergent cases 


\section{Cureus}

Over the 34-month study period (January 1, 2015-October 31, 2017), trauma surgery (24\%) and neurosurgery (18\%) comprised the most Level 1 cases (Figure 2), with transplant (19\%) and trauma surgery (17\%) comprising the most Level 2 cases (Figure 3). General surgery and orthopaedic surgery represented the majority of Level 3-Level 5 cases (Figures 4-6).

\section{Level 1}

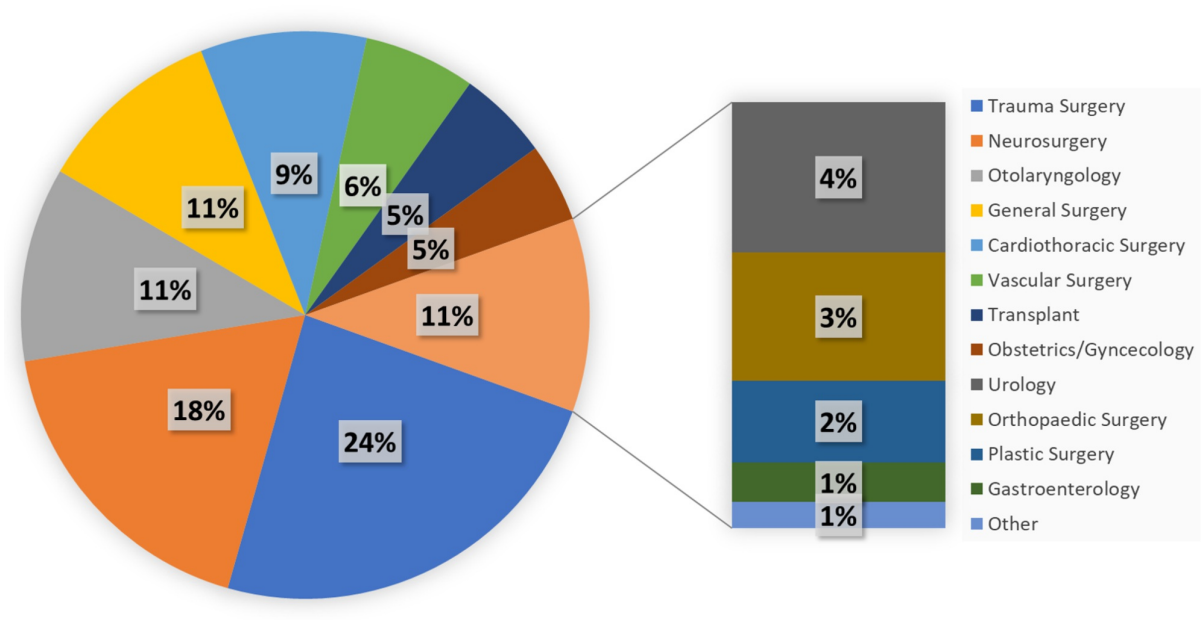

FIGURE 2: Breakdown of Level 1 cases by service.

Breakdown of surgical cases posted as Level 1 by primary service.

\section{Level 2}

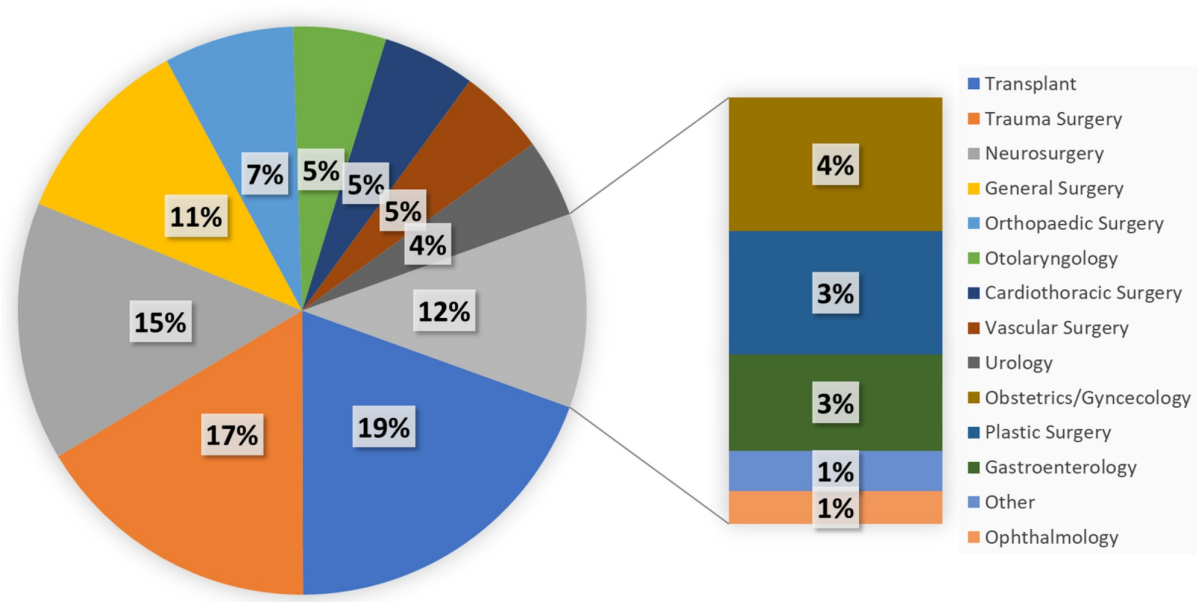

FIGURE 3: Breakdown of Level 2 cases by service.

Breakdown of surgical cases posted as Level 2 by primary service. 


\section{Cureus}

Level 3

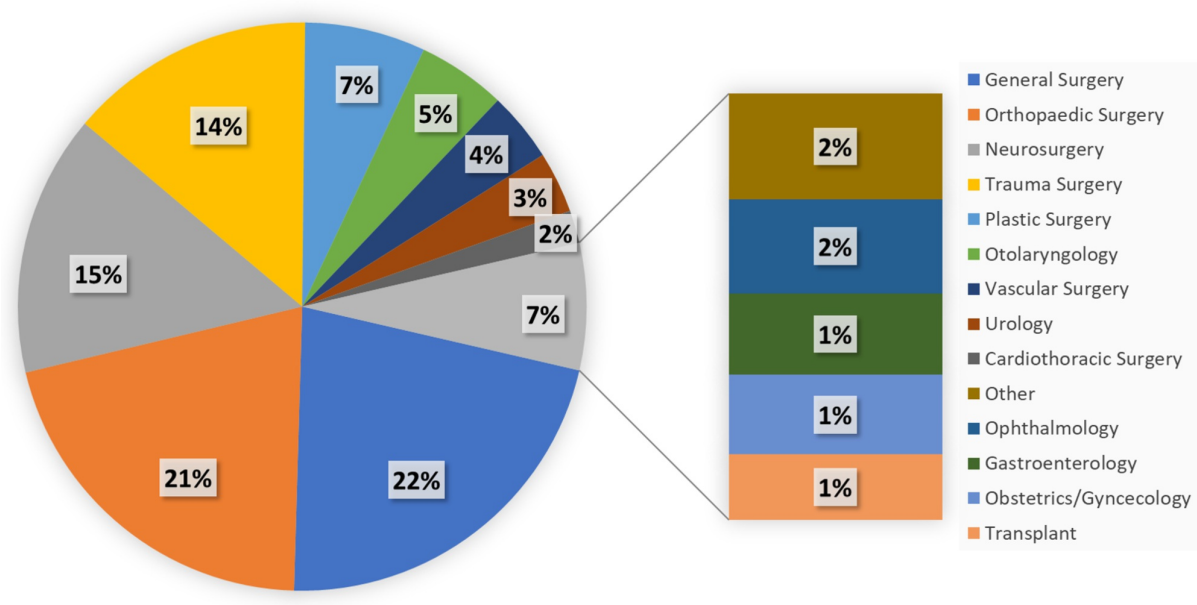

FIGURE 4: Breakdown of Level 3 cases by service.

Breakdown of surgical cases posted as Level 3 by primary service.

\section{Level 4}

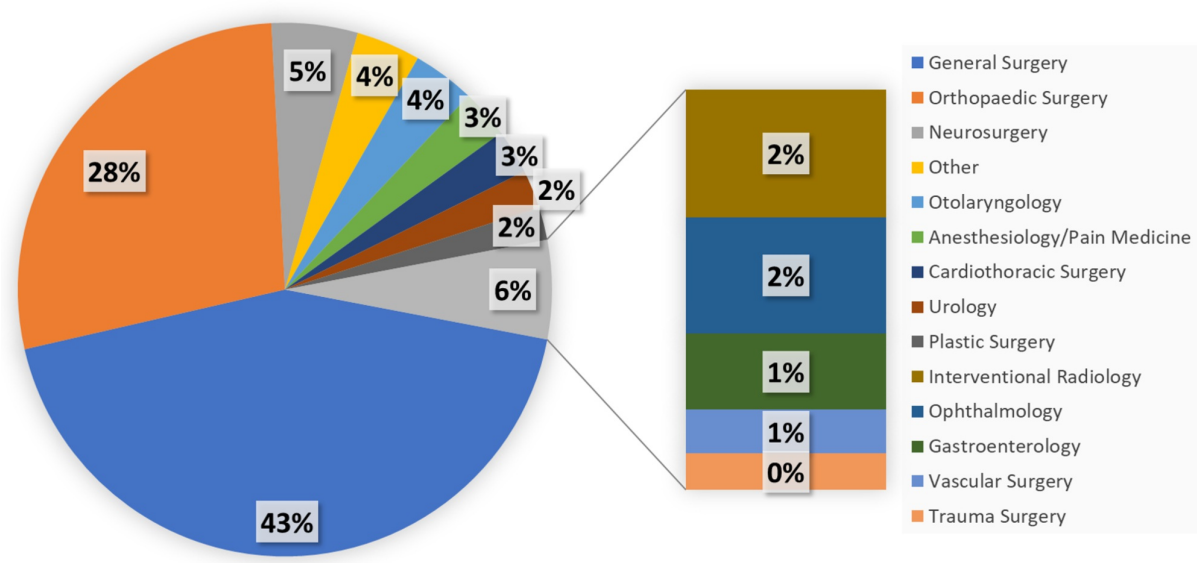

FIGURE 5: Breakdown of Level 4 cases by service.

Breakdown of surgical cases posted as Level 4 by primary service. 


\section{Cureus}

\section{Level 5}

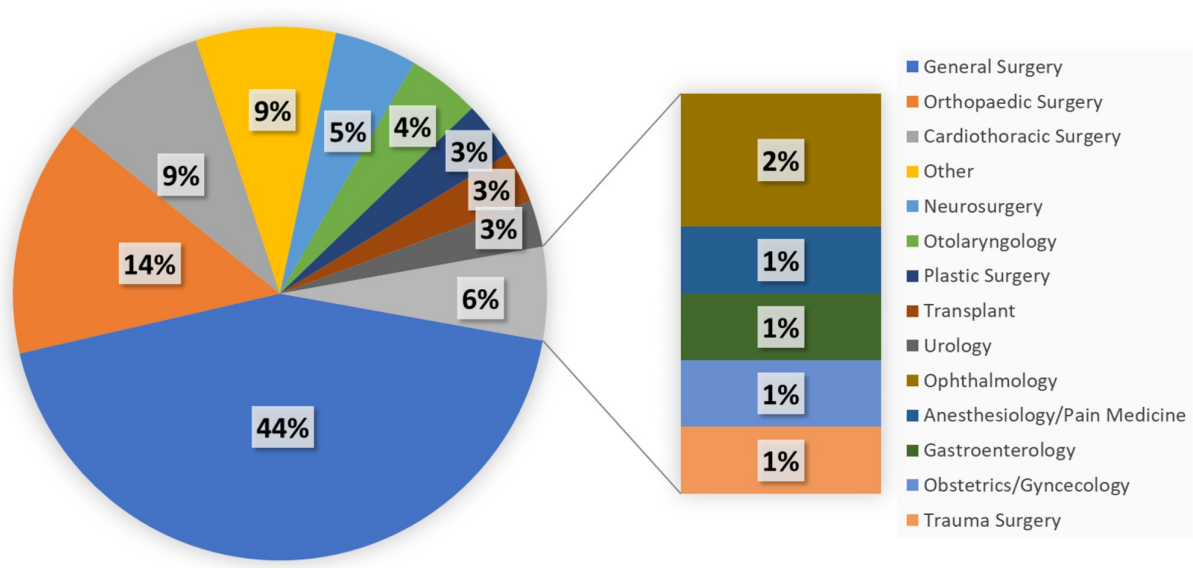

\section{FIGURE 6: Breakdown of Level 5 cases by service.}

Breakdown of surgical cases posted as Level 5 by primary service.

Among trauma surgery, exploratory laparotomy, appendectomy, and abdominal wound revision/irrigation/exploration/debridement were the most common urgent/emergent cases. Similarly, exploratory laparotomy, appendectomy, and extracorporeal membrane oxygenation (ECMO) cannula placement were the most common general surgery cases. Ventriculoperitoneal (VP) shunt placement, craniotomy for epidural hematoma, and VP shunt revision were the most common neurosurgery cases. Open reduction of the elbow, wound revision/irrigation/exploration/debridement, and open reduction of the femur were the most common orthopaedic surgery cases (Tables 2, 3).

\begin{tabular}{|c|c|c|c|}
\hline Department & Procedure Category & Number & $\begin{array}{l}\text { Percentage of All } \\
\text { Cases }\end{array}$ \\
\hline \multirow{3}{*}{$\begin{array}{l}\text { Anesthesiology/Pain } \\
\text { Medicine }\end{array}$} & PICC Line Insertion & 46 & $75.4 \%$ \\
\hline & Intubation & 7 & $11.5 \%$ \\
\hline & Extubation & 3 & $4.9 \%$ \\
\hline \multirow{4}{*}{ Cardiothoracıc Surgery } & Wound Revision/Irrigation/Exploration/Debridement & 114 & $20.4 \%$ \\
\hline & ECMO Cannulation & 74 & $13.2 \%$ \\
\hline & Aorta Repair & 44 & $7.9 \%$ \\
\hline & Lung Transplant & 44 & $7.9 \%$ \\
\hline \multirow{3}{*}{ Gastroenterology } & Endoscopic Retrograde Cholangiopancreatography & 96 & $52.1 \%$ \\
\hline & Esophagogastroduodenoscopy & 55 & $29.9 \%$ \\
\hline & Endoscopy-Upper Gl & 10 & $5.4 \%$ \\
\hline \multirow{4}{*}{ General Surgery } & Exploratory Laparotomy & 278 & $12.8 \%$ \\
\hline & Appendectomy & $2 / 3$ & $12.5 \%$ \\
\hline & ECMO Cannulation & 96 & $4.4 \%$ \\
\hline & GJ Tube Placement & 27 & $57.4 \%$ \\
\hline
\end{tabular}




\section{Cureus}

\begin{tabular}{|c|c|c|c|}
\hline \multirow[t]{2}{*}{ Interventional Radiology } & G/GJ Tube Removal & 9 & $19.1 \%$ \\
\hline & G Tube Placement & 4 & $8.5 \%$ \\
\hline \multirow{3}{*}{ Neurosurgery } & VP Shunt Placement & 256 & $16.6 \%$ \\
\hline & Craniotomy for Hematoma & 148 & $9.6 \%$ \\
\hline & VP Shunt Revision & 229 & $9.4 \%$ \\
\hline \multirow{3}{*}{ Obstetrics/Gynecology } & Salpingectomy/Salpingo-oophorectomy & 54 & $18.7 \%$ \\
\hline & Uterine Dilatation and Curettage & 50 & $17.3 \%$ \\
\hline & Exploratory Laparoscopy & 39 & $13.5 \%$ \\
\hline \multirow{3}{*}{ Ophthalmology } & Globe Repair & 42 & $35.6 \%$ \\
\hline & Blepharoplasty & 15 & $12.7 \%$ \\
\hline & Vitrectomy & 15 & $12.7 \%$ \\
\hline \multirow{3}{*}{ Orthopaedic Surgery } & Open Reduction-Elbow & 230 & $15.6 \%$ \\
\hline & Wound Revision/Irrigation/Exploration/Debridement & 104 & $7.0 \%$ \\
\hline & Open Reduction-Femur & 72 & $4.9 \%$ \\
\hline \multirow{3}{*}{ Otolaryngology } & Wound Revision/Irrigation/Exploration/Debridement & 123 & $17.7 \%$ \\
\hline & Tracheostomy & 81 & $11.7 \%$ \\
\hline & Bronchoscopy & 79 & $11.4 \%$ \\
\hline \multirow{3}{*}{ Plastic Surgery } & Open Reduction-Mandible & 54 & $12.9 \%$ \\
\hline & $\begin{array}{l}\text { Wound Revision/lrrigation/ Exploration/Debridement - Chest } \\
\text { Wall }\end{array}$ & 38 & $9.1 \%$ \\
\hline & Wound Revision/Irrigation/Debridement - Hand & 25 & $6.0 \%$ \\
\hline \multirow{3}{*}{$\begin{array}{l}\text { Transplant/Abdominal } \\
\text { Surgery }\end{array}$} & Kidney Transplant & 367 & $39.8 \%$ \\
\hline & Liver Transplant & 213 & $23.1 \%$ \\
\hline & Exploratory Laparotomy & 117 & $12.7 \%$ \\
\hline \multirow{3}{*}{ Trauma Surgery } & Exploratory Laparotomy & 455 & $27.9 \%$ \\
\hline & Appendectomy & 186 & $11.4 \%$ \\
\hline & Wound Revision//rrigation/Exploration/Debridement - Abdomen & 164 & $10.1 \%$ \\
\hline \multirow{3}{*}{ Urology } & Ureteral Stent Placement & 115 & $27.7 \%$ \\
\hline & Cystoscopy & 45 & $10.8 \%$ \\
\hline & Wound Revision/ Irrigation/Exploration/Debridement & 32 & $7.7 \%$ \\
\hline \multirow{3}{*}{ Vascular Surgery } & Toe Amputation & 81 & $17.1 \%$ \\
\hline & Wound Revision/Irrigation/Exploration/Debridement - Foot & 67 & $14.1 \%$ \\
\hline & Wound Revision/Irrigation/Exploration/Debridement - Leg & 29 & $6.1 \%$ \\
\hline \multirow{3}{*}{ Other } & Bone Marrow Biopsy & 66 & $33.0 \%$ \\
\hline & Bronchoscopy & 25 & $12.5 \%$ \\
\hline & Lumbar Puncture & 17 & 8.5 \\
\hline
\end{tabular}

TABLE 2: Most common procedure by department for all leveled cases at the Johns Hopkins Hospital, 2015-2017.

PICC: Peripherally inserted central catheter; ECMO: Extracorporeal membrane oxygenation; GI: Gastrointestinal; GJ: Gastrojejunostomy; VP: Ventriculoperitoneal. 


\section{Cureus}

\begin{tabular}{|c|c|c|c|}
\hline Department & Level & Procedure Category & $\begin{array}{l}\text { Percentage of Department Cases at } \\
\text { this Level }\end{array}$ \\
\hline \multirow{6}{*}{$\begin{array}{l}\text { Anesthesiology/Pain } \\
\text { Medicine }\end{array}$} & 1 & Intubation & $100 \%$ \\
\hline & & Intubation & $50.0 \%$ \\
\hline & & Intrathecal Pump Revision & $50.0 \%$ \\
\hline & 3 & PICC Line Insertion & $75.0 \%$ \\
\hline & 4 & PICC Line Insertion & $85.7 \%$ \\
\hline & 5 & Three Different Procedures & $33.3 \%$ \\
\hline \multirow{5}{*}{ Cardiothoracic Surgery } & 1 & Wound Revision - Thorax & $27.2 \%$ \\
\hline & 2 & Lung Transplant & $21.6 \%$ \\
\hline & 3 & \multirow{3}{*}{ Wound Revision - Thorax } & $20.4 \%$ \\
\hline & 4 & & $33.3 \%$ \\
\hline & 5 & & $20.7 \%$ \\
\hline \multirow{5}{*}{ Gastroenterology } & 1 & \multirow{2}{*}{ Endoscopic Retrograde Cholangiopancreatography } & $56.0 \%$ \\
\hline & 2 & & $67.0 \%$ \\
\hline & 3 & & $45.0 \%$ \\
\hline & 4 & & $63.2 \%$ \\
\hline & 5 & Three Different Procedures & $33.3 \%$ \\
\hline \multirow{5}{*}{ General Surgery } & 1 & \multirow{2}{*}{ Exploratory Laparotomy } & $41.6 \%$ \\
\hline & 2 & & $25.9 \%$ \\
\hline & 3 & & $21.8 \%$ \\
\hline & 4 & & $14.5 \%$ \\
\hline & 5 & Hickman Catheter Placement & $7.2 \%$ \\
\hline \multirow{5}{*}{ Interventional Radiology } & 1 & Angiogram & $100 \%$ \\
\hline & 2 & Three Different Procedures & $33.3 \%$ \\
\hline & 3 & \multirow{3}{*}{ GJ Tube Placement } & $63.6 \%$ \\
\hline & 4 & & $62.5 \%$ \\
\hline & 5 & & $57.4 \%$ \\
\hline \multirow{5}{*}{ Neurosurgery } & 1 & Craniotomy for Hematoma & $19.4 \%$ \\
\hline & 2 & \multirow{4}{*}{ VP Shunt Placement } & $15.3 \%$ \\
\hline & 3 & & $20.9 \%$ \\
\hline & 4 & & $31.0 \%$ \\
\hline & 5 & & $25.0 \%$ \\
\hline \multirow{5}{*}{ Obstetrics/Gynecology } & 1 & Exploratory Laparoscopy & $20.4 \%$ \\
\hline & \multirow{2}{*}{2} & Salpingectomy & $21.6 \%$ \\
\hline & & \multirow{2}{*}{ Dilatation and Curettage - Uterus } & $21.6 \%$ \\
\hline & 3 & & $17.9 \%$ \\
\hline & 4 & Five Different Procedures & $20.0 \%$ \\
\hline
\end{tabular}




\section{Cureus}

\begin{tabular}{|c|c|c|c|}
\hline & 5 & $\begin{array}{l}\text { Wound Revision/Irrigation/Exploration/Debridement - } \\
\text { Abdomen }\end{array}$ & $66.7 \%$ \\
\hline \multirow{5}{*}{ Ophthalmology } & 1 & Vitrectomy & $50.0 \%$ \\
\hline & 2 & \multirow{3}{*}{ Globe Repair } & $45.5 \%$ \\
\hline & 3 & & $37.0 \%$ \\
\hline & 4 & & $31.0 \%$ \\
\hline & 5 & Examination Under Anesthesia - Eye & $33.3 \%$ \\
\hline \multirow{5}{*}{ Orthopaedic Surgery } & 1 & Fasciotomy & $41.3 \%$ \\
\hline & 2 & Wound Revision/Irrigation/Exploration/Debridement - Leg & $11.5 \%$ \\
\hline & 3 & \multirow{3}{*}{ Open Reduction - Elbow } & $16.3 \%$ \\
\hline & 4 & & $23.1 \%$ \\
\hline & 5 & & $15.2 \%$ \\
\hline \multirow{5}{*}{ Otolaryngology } & 1 & Tracheostomy & $18.4 \%$ \\
\hline & 2 & & $15.6 \%$ \\
\hline & 3 & $\begin{array}{l}\text { Wound Revision/Irrigation/Exploration/Debridement - } \\
\text { Neck }\end{array}$ & $16.4 \%$ \\
\hline & 4 & & $25.4 \%$ \\
\hline & 5 & Bronchoscopy & $35.7 \%$ \\
\hline \multirow{4}{*}{ Plastic Surgery } & 1 & $\begin{array}{l}\text { Wound Revision/Irrigation/Exploration/Debridement - } \\
\text { Chest Wall/Breast }\end{array}$ & $17.6 \%$ \\
\hline & 2 & Open Reduction - Mandible & $13.7 \%$ \\
\hline & 4 & Wound Revision/Irrigation/Exploration/Debridement - Leg & $18.2 \%$ \\
\hline & 5 & Cranioplasty & $27.3 \%$ \\
\hline \multirow{5}{*}{$\begin{array}{l}\text { Transplant/Abdominal } \\
\text { Surgery }\end{array}$} & 1 & Exploratory Laparotomy & $51.2 \%$ \\
\hline & 2 & Kidney Transplant & $46.6 \%$ \\
\hline & 3 & $\begin{array}{l}\text { Wound Revision/Irrigation/Exploration/Debridement - } \\
\text { Abdomen }\end{array}$ & $46.9 \%$ \\
\hline & 4 & Three Different Procedures & $33.3 \%$ \\
\hline & 5 & Kidney Transplant & $40.0 \%$ \\
\hline \multirow{5}{*}{ Trauma Surgery } & 1 & Exploratory Laparotomy & $\begin{array}{l}52.1 \% \\
22.3 \%\end{array}$ \\
\hline & 3 & Appendectomy & $25.1 \%$ \\
\hline & & Appendectomy & $22.2 \%$ \\
\hline & 4 & $\begin{array}{l}\text { Wound Revision/Irrigation/Exploration/Debridement - } \\
\text { Foot }\end{array}$ & $22.2 \%$ \\
\hline & 5 & Three Different Procedures & $33.3 \%$ \\
\hline \multirow{5}{*}{ Urology } & 1 & Orchiopexy & $26.6 \%$ \\
\hline & 2 & Ureteral Stent Placement & $39.0 \%$ \\
\hline & 3 & Ureteral Stent Placement & $32.7 \%$ \\
\hline & 4 & Cystoscopy & $28.2 \%$ \\
\hline & 5 & Cystoscopy & $22.2 \%$ \\
\hline
\end{tabular}




\section{Cureus}

\begin{tabular}{|c|c|c|c|}
\hline \multirow{5}{*}{ Vascular Surgery } & 1 & Thrombectomy-Unspecified Vessel & $10.5 \%$ \\
\hline & 2 & $\begin{array}{l}\text { Wound Revision/Irrigation/Exploration/Debridement - } \\
\text { Foot }\end{array}$ & $23.3 \%$ \\
\hline & 3 & Amputation - Toe & $30.4 \%$ \\
\hline & 4 & $\begin{array}{l}\text { Wound Revision/Irrigation/Exploration/Debridement - } \\
\text { Foot }\end{array}$ & $27.3 \%$ \\
\hline & 5 & Fasciotomy & $100 \%$ \\
\hline \multirow{5}{*}{ Other } & 1 & Bronchoscopy & $25.0 \%$ \\
\hline & 2 & $\begin{array}{l}\text { Wound Revision/Irrigation/Exploration/Debridement - } \\
\text { Chest Wall/Breast }\end{array}$ & $20.0 \%$ \\
\hline & 3 & \multirow{3}{*}{ Bone Marrow Biopsy } & $26.9 \%$ \\
\hline & 4 & & $50.8 \%$ \\
\hline & 5 & & $66.7 \%$ \\
\hline
\end{tabular}

TABLE 3: Most common procedure type by level and listing department for all leveled cases at the Johns Hopkins Hospital, 2015-2017.

PICC: Peripherally inserted central catheter; VP: Ventriculoperitoneal; GJ: Gastrojejunostomy.

When assessed by level of urgency, exploratory laparotomy was the most common Level 1 procedure and second most common Level 2 procedure. ECMO cannulation and craniotomy for hematoma evacuation were the next most common Level 1 procedures. Kidney transplant, exploratory laparotomy, and abdominal wound revision/irrigation/exploration/debridement were the most common Level 2 procedures. Appendectomy, open reduction of the elbow, leg wound revision/irrigation/exploration/debridement, and esophagogastroduodenoscopy (EGD) were the most common Level 3 and Level 4 cases. The most common Level 5 cases were bone marrow biopsy, bronchoscopy, and Hickman catheter placement (Table 4). 


\section{Cureus}

\begin{tabular}{|c|c|c|c|}
\hline Level & Procedure Type & Number of Cases & Proportion of All Cases of this Level \\
\hline \multirow{3}{*}{1} & Exploratory Laparotomy & 515 & $21.3 \%$ \\
\hline & ECMO Cannulation & 108 & $4.5 \%$ \\
\hline & Craniotomy for Hematoma Evacuation & 84 & $3.5 \%$ \\
\hline \multirow{3}{*}{2} & Kidney Transplant & 351 & $9.1 \%$ \\
\hline & Exploratory Laparotomy & 326 & $8.4 \%$ \\
\hline & Wound Revision/Irrigation/Exploration/Debridement - Abdomen & 232 & $6.0 \%$ \\
\hline \multirow{3}{*}{3} & Appendectomy & 241 & $8.3 \%$ \\
\hline & Open Reduction - Elbow & 98 & $3.4 \%$ \\
\hline & Wound Revision/Irrigation/Exploration/Debridement - Leg & 96 & $3.3 \%$ \\
\hline \multirow{3}{*}{4} & Appendectomy & 107 & $6.4 \%$ \\
\hline & Open Reduction - Elbow & 107 & $6.4 \%$ \\
\hline & Esophagogastroduodenoscopy & 53 & $3.2 \%$ \\
\hline \multirow{3}{*}{5} & Bone Marrow Biopsy & 18 & $5.6 \%$ \\
\hline & Bronchoscopy & 13 & $4.1 \%$ \\
\hline & Hickman Catheter Placement & 10 & $3.1 \%$ \\
\hline \multirow{3}{*}{ Any } & Exploratory Laparotomy & 925 & $8.3 \%$ \\
\hline & Appendectomy & 462 & $4.1 \%$ \\
\hline & Wound Revision//rrigation/Exploration/Debridement & 396 & $3.5 \%$ \\
\hline
\end{tabular}

TABLE 4: Most common procedures by level at the Johns Hopkins Hospital, 2015-2017.

ECMO: Extracorporeal membrane oxygenation

\section{Operating room waiting time}

Over the 34-month period, the waiting time between the posting of an urgent/emergent case to when it entered the operating room (post-to-room time) decreased significantly over each year $(\mathrm{p}<0.05)$. The mean post-to-room times from 2015, 2016, and 2017 were $193.40 \pm 4.78,177.20 \pm 3.29$, and $82.01 \pm 2.98$, respectively (Table 5). Chi-square previously demonstrated no significant differences with regards to the proportion of each level represented, among all urgent/emergent cases for a given year. Given the consistent distribution of urgency over the three years, it is unlikely that differences in post-to-room time be attributed to greater proportions of Level 1 and Level 2 cases in later years. 


\section{Cureus}

\begin{tabular}{|c|c|c|c|c|c|}
\hline & 2015 & 2016 & $2017^{*}$ & \multicolumn{2}{|l|}{ Overall } \\
\hline \multicolumn{6}{|c|}{ Post-to-Room Time } \\
\hline $\mathrm{N}$ & 4016 & 3892 & 3298 & \multicolumn{2}{|l|}{11206} \\
\hline Mean Time (min) & $193.40 \pm 4.78$ & $177.20 \pm 3.29$ & $82.01 \pm 2.98$ & \multicolumn{2}{|l|}{$139.40 \pm 2.23$} \\
\hline \multicolumn{6}{|l|}{ Case Duration } \\
\hline $\mathrm{N}$ & 4016 & 3892 & 3298 & \multicolumn{2}{|l|}{11206} \\
\hline \multirow[t]{2}{*}{ Mean Time (min) } & $158.16 \pm 1.98$ & $158.07 \pm 1.88$ & $164.27 \pm 2.90$ & \multicolumn{2}{|l|}{$159.97 \pm 1.29$} \\
\hline & Level 1 & Level 2 & Level 3 & Level 4 & Level 5 \\
\hline \multicolumn{6}{|c|}{ Post-to-Room Time } \\
\hline $\mathrm{N}$ & 2419 & 3879 & 2912 & 1676 & 320 \\
\hline Mean Time (min) & $31.22 \pm 3.42$ & $148.99 \pm 4.40$ & $145.17 \pm 2.45$ & $180.44 \pm 4.43$ & $573.45 \pm 25.88$ \\
\hline \multicolumn{6}{|l|}{ Case Duration } \\
\hline $\mathrm{N}$ & 2419 & 3879 & 2912 & 1676 & 320 \\
\hline Mean Time (min) & $169.46 \pm 2.71$ & $200.29 \pm 2.57$ & $136.28 \pm 2.16$ & $101.58 \pm 1.89$ & $125.70 \pm 4.85$ \\
\hline
\end{tabular}

TABLE 5: Post-to-room time and case duration of procedures by year.

The mean overall post-to-room time for Level 1 cases, $31.22 \pm 3.42$ minutes, was significantly below the threshold one hour acceptable waiting time $(\mathrm{p}<0.05)$. When assessed by department, orthopaedic surgery was the only service where the mean post-to-room time exceeded the allowable one hour for Level 1 cases (105.2 \pm 69.9 minutes), with a violation frequency present in $9 \%$ (7/80) of orthopaedic Level 1 cases. Among Level 2 cases, Chi-square demonstrated that the frequency of post-to-room time violations, exceeding the two-hour allowable period, was only significant for transplant surgery (mean $297.7 \pm 35.9$ minutes) and cardiothoracic surgery (mean $190 \pm 21.5$ minutes) $(p<0.05$ for both; $p>0.05$ for all other departments). All other departments had a mean post-to-room time within the two-hour allowable period, with a frequency of violation that was not significant on Chi-square analysis ( $p>0.05$ for all). The overall mean post-to-room time was within the allowable time for Level $3-5$ cases, as were the mean post-to-room times with respect to department ( $p>0.05$ for all). The frequency of post-to-room time violations, with respect to level and department, was not significant for any department among Level $3-5$ cases $(p<0.05$ for all) (Table 5).

\section{Discussion}

Trauma system regionalization of patients with life-threatening emergent and urgent cases to Level 1 trauma centers has demonstrated significant reduction in hospital mortality [10]. Among Level 1 trauma centers, however, there is a lack of established triage protocols to optimize surgical timing. Triage protocols for urgent and emergent operative cases reported in the literature typically focus on general surgery aloneunderrepresent the variety of surgical specialties present in a Level 1 trauma center [2,3,7-10]. Additionally, there are few studies demonstrating the implementation and efficacy of such protocols at large-volume centers.

Following a Delphi method of international expert opinions and questionnaires, the World Society for Emergency Surgery (WSES) created a standard triage protocol known as the Timing of Acute Care Surgery classification (TACS) [9]. However, there are no reports describing the implementation or efficacy of this classification system. In a nationwide cohort study of 173,643 general surgery cases, by Mullen et al. [13], laparoscopic cholecystectomy and laparoscopic appendectomy were the most common urgent and emergent surgical cases. However, the study failed to compare the frequency of urgent and emergent cases from other surgical specialties, with respect to institution and study period. In addition, there was a heterogenous population of institutions represented, without stratification for Level 1 trauma centers. In the current study of 11,209 cases, exploratory laparotomy, ECMO cannulation, and craniotomy for epidural hematoma were the most common Level 1 cases, performed by the respective departments of trauma surgery, general surgery, and neurosurgery.

Some authors have suggested the use of dedicated ORs for emergency surgery. However, this is often not feasible or efficient in large volume centers, and has shown mixed results with respect to waiting time $[14,15]$. In a study implementing dedicated operating rooms for emergency surgery, from a large children's 
Level 1 trauma hospital, dedicated 'add-on' ORs resulted in decreased elective surgery cancellations but did not significantly impact waiting times for emergency cases designated Priority 1 ( $\leqslant 1 \mathrm{hr}$ ) or Priority $2(\leqslant 4 \mathrm{hr})$ [15]. It is important to note here that the current institution has two ORs designated as trauma rooms, into which urgent/emergent cases frequently are placed. However, there is no precedent of always having an OR empty and waiting for an emergency as exists at some trauma centers.

Several authors have proposed mathematical algorithms to inform sequencing of urgent/emergent cases [16-18]. In one such model, Dexter et al. [18] summarize three objectives when scheduling emergent operative cases: 1) minimizing wait time, 2) adhering to the posting order, and 3) reflecting medical priority. The protocol implemented at Johns Hopkins Hospital exemplifies these three objectives. Additionally, the mean overall waiting period for an urgent/emergent case entering the OR decreased significantly each year ( $\mathrm{p}$ 0.05), resulting in a waiting time that was less than half from 2015 to 2017 (193 vs. 82 minutes). This was accomplished without any significant change in the distribution of urgency between each year. As such, these results suggest that acclimation and multi-departmental practice with an established protocol is necessary in order to match clinically acceptable waiting times for urgent/emergent cases.

Limitations of this study include those inherent to retrospective single-institution studies. The study is also limited in reporting clinical outcomes following implementation of the trauma protocol. We acknowledge that the distribution of urgent/emergent cases may vary from institution to institution, depending on the referral region, relative size of various departments, and other factors. However, to the authors' knowledge, this is the first study to characterize all urgent and emergent cases at a large academic Level 1 trauma center, outline the specialty and nature of emergent operative cases, and assess the efficacy of the institutional trauma protocol on surgical waiting times. We hope this description of types of urgent/emergent cases and validation of our institution's protocol for reducing OR waiting time will be helpful to other large-volume Level 1 trauma centers.

\section{Conclusions}

Level 1 trauma centers are capable of caring for every aspect of injury and have 24-hour in-house coverage by general surgeons, with prompt availability of orthopedic surgery, neurosurgery, anesthesiology, emergency medicine, radiology, internal medicine, plastic surgery, oral and maxillofacial surgery, pediatric and critical care. Despite the wide variety of trauma, protocols in the current literature often focus on a single surgical service. To the authors' knowledge, this is the first study to characterize all urgent and emergent cases at a large academic Level 1 trauma center across all surgical specialties, to outline the specialty and nature of emergent operative cases, and to assess the efficacy of the institutional trauma protocol on surgical waiting times over a 34-month period.

\section{Additional Information \\ Disclosures}

Human subjects: Consent was obtained by all participants in this study. N/A issued approval N/A. This study was IRB exempt because no patient-specific PHI was obtained for this study. The data was collected as part of quality improvement purposes. Animal subjects: All authors have confirmed that this study did not involve animal subjects or tissue. Conflicts of interest: In compliance with the ICMJE uniform disclosure form, all authors declare the following: Payment/services info: All authors have declared that no financial support was received from any organization for the submitted work. Financial relationships: Corinna Zygourakis, Nicholas Theodore declare(s) Consultant from SpineAlign. Consultant. Nicholas Theodore declare(s) a patent, royalties and stock/stock options from Globus. Camilo Molina declare(s) personal fees from Augmedics. Consultant. Other relationships: All authors have declared that there are no other relationships or activities that could appear to have influenced the submitted work.

\section{References}

1. Trauma center levels explained. (2017). Accessed: November 25, 2017: https://www.amtrauma.org/page/traumalevels.

2. Catena F, Moore EE: World Journal of Emergency Surgery (WJES), World Society of Emergency Surgery (WSES) and the role of emergency surgery in the world. World J Emerg Surg. 2007, 2:3. 10.1186/1749-79222-3

3. Eko FN, Ryb GE, Drager L, Goldwater E, Wu JJ, Counihan TC: Ideal timing of surgery for acute uncomplicated appendicitis. N Am J Med Sci. 2013, 5:22-27. 10.4103/1947-2714.106186

4. Stanislavovych SV, Vasylivna KU: Choice of optimal time and type of orthopedic surgery in multiple injured patients with acute respiratory distress syndrome (ARDS) depending on age: retrospective study. Ortop Traumatol Rehabil. 2013, 15:335-340. 10.5604/15093492.1073832

5. McIsaac DI, Abdulla K, Yang H, et al.: Association of delay of urgent or emergency surgery with mortality and use of health care resources: a propensity score-matched observational cohort study. CMAJ. 2017, 189:E905-E912. 10.1503/cmaj.160576

6. Cosgrove JF, Gaughan M, Snowden CP, Lees T: Decreasing delays in urgent and expedited surgery in a university teaching hospital through audit and communication between peri-operative and surgical directorates. Anaesthesia. 2008, 63:599-603. 10.1111/j.1365-2044.2008.05441.x

7. O’Leary DP, Beecher S, McLaughlin R: Emergency surgery pre-operative delays - realities and economic 


\section{Cureus}

impacts. Int J Surg. 2014, 12:1333-1336. 10.1016/j.ijsu.2014.10.002

8. Stepaniak PS, Dexter F: Constraints on the scheduling of urgent and emergency surgical cases: surgeon, equipment, and anesthesiologist availability. Perioper Care Oper Room Manag. 2016, 3:6-11.

10.1016/j.pcorm.2016.02.001

9. Kluger Y, Ben-Ishay O, Sartelli M, et al.: World society of emergency surgery study group initiative on Timing of Acute Care Surgery classification (TACS). World J Emerg Surg. 2013, 8:E17. 10.1186/1749-7922-817

10. Schechtman D, He JC, Zosa BM, Allen D, Claridge JA: Trauma system regionalization improves mortality in patients requiring trauma laparotomy. J Trauma Acute Care Surg. 2017, 82:58-64.

10.1097/TA.0000000000001302

11. McManus ML, Long MC, Cooper A, Mandell J, Berwick DM, Pagano M, Litvak E: Variability in surgical caseload and access to intensive care services. Anesthesiology. 2003, 98:1491-1496.

12. McManus ML, Long MC, Cooper A, Litvak E: Queuing theory accurately models the need for critical care resources. Anesthesiology. 2004, 100:1271-1276.

13. Mullen MG, Michaels AD, Mehaffey JH, Guidry CA, Turrentine FE, Hedrick TL, Friel CM: Risk associated with complications and mortality after urgent surgery vs elective and emergency surgery. JAMA Surg. 2017, 152:768-774. 10.1001/jamasurg.2017.0918

14. van Veen-Berkx E, Elkhuizen SG, Kuijper B, Kazemier G： Dedicated operating room for emergency surgery generates more utilization, less overtime, and less cancellations. Am J Surg. 2016, 211:122-128. 10.1016/j.amjsurg.2015.06.021

15. Heng M, Wright JG: Dedicated operating room for emergency surgery improves access and efficiency. Can J Surg. 2013, 56:167-174. 10.1503/cjs.019711

16. Lin D, Patrick J, Labeau F: Estimating the waiting time of multi-priority emergency patients with downstream blocking. Health Care Manag Sci. 2014, 17:88-99. 10.1007/s10729-013-9241-3

17. Torkki PM, Alho AI, Peltokorpi AV, Torkki MI, Kallio PE: Managing urgent surgery as a process: case study of a trauma center. Int J Technol Assess Health Care. 2006, 22:255-260. 10.1017/S0266462306051087

18. Dexter F, Macario A, Traub RD: Optimal sequencing of urgent surgical cases. Scheduling cases using operating room information systems. J Clin Monit Comput. 1999, 15:153-162. 10.1023/A:1009941214632 